\title{
Association between potassium channel SNPs and essential hypertension in Xinjiang Kazak Chinese patients
}

\author{
YUAN-YUAN HAN $^{1 *}$, LI-JIE WANG ${ }^{1 *}$, LIANG ZHANG $^{1,2}$, WEN-WEN ZHANG ${ }^{1}$, \\ KE-TAO MA ${ }^{1,2}, \mathrm{LI} \mathrm{LI}^{1,2}$ and JUN-QIANG SI ${ }^{1-4}$ \\ ${ }^{1}$ Department of Physiology; ${ }^{2}$ The Key Laboratory of Xinjiang Endemic and Ethnic Diseases, \\ Medical College of Shihezi University, Shihezi, Xinjiang 832002; ${ }^{3}$ Department of Physiology, \\ Huazhong University of Science and Technology of Basic Medical Sciences; ${ }^{4}$ Department of Physiology, \\ Wuhan University School of Basic Medical Sciences, Wuhan, Hubei 430070, P.R. China
}

Received July 25, 2016; Accepted May 5, 2017

DOI: $10.3892 / \mathrm{etm} .2017 .4734$

\begin{abstract}
The aim of the present study was to examine whether single-nucleotide polymorphisms (SNPs) of $\beta 1$ subunit of large-conductance $\mathrm{Ca}^{2+}$-activated $\mathrm{K}^{+}$channel (KCNMB1) and inwardly rectifying $\mathrm{K}^{+}$channel, subfamily $\mathrm{J}$, member-11 (KCNJ11) are associated with essential hypertension (EH) in Xinjiang Kazak Chinese patients. A polymerase chain reaction-restriction fragment length polymorphism technique was applied to detect the distribution of selected alleles and genotype frequencies in a cohort of Xinjiang Kazak Chinese patients. Samples from 267 patients with EH and 259 normotensive (NT) controls were analyzed. An unconditional logistic regression analysis was used to estimate the odds ratio and $95 \%$ confidence interval of the risk factors that are associated with the development of EH. Genotype and allele frequency analyses revealed that the frequency of genotypes KCNJ11-rs2285676 and KCNMB1-rs11739136 was not significantly different between the EH and NT groups. Individuals carrying the GG genotype of KCNJ11-rs5219 had a 2.08 times higher risk of having $\mathrm{EH}$ than individuals carrying the GA+AA genotype of KCNJ11-rs5219. Furthermore, the $\mathrm{G}$ allele frequency of KCNJ11-rs5219 in the EH group was significantly higher than that of the NT group $(\mathrm{P}=0.048)$. Additionally, logistic regression analysis revealed that the body weight and GG genotype of KCNJ11-rs5219 were positively associated with EH in Xinjiang Kazak Chinese patients $(\mathrm{P}<0.01)$
\end{abstract}

Correspondence to: Dr Jun-Qiang $\mathrm{Si}$ or Dr Liang Zhang, Department of Physiology, Medical College of Shihezi University, 59 North 2nd Road, Shihezi, Xinjiang 832002, P.R. China

E-mail: sijunqiang@shzu.edu.cn

E-mail: zhangliang_0622@163.com

*Contributed equally

Key words: essential hypertension, inward rectifier $\mathrm{K}^{+}$channel, single-nucleotide polymorphism

\section{Introduction}

Essential hypertension (EH) is a complex polygenic hereditary disease caused by various genetic and environmental factors (1). In total, $25-40 \%$ of the adult population are hypertensive, and $>90 \%$ of cases are of unknown origin, which are defined as EH (2). An increase of vascular tone in resistance arteries is the basic pathophysiological mechanism of EH (3-5). The large-conductance $\mathrm{Ca}^{2+}$-activated $\mathrm{K}^{+}\left(\mathrm{BK}_{\mathrm{Ca}}\right)$ channel, which is composed of an ion-conducting (pore forming) $\alpha$-subunit and regulatory $\beta$-subunit, acts as a negative feedback in the control of vascular tone and blood pressure $(6,7)$.

The $\beta 1$-regulatory subunit of the $\mathrm{BK}_{\mathrm{Ca}}$ channel is mainly expressed in vascular smooth muscle cells (VSMCs), which is encoded by the large conductance, voltage and $\mathrm{Ca}^{2+}$-sensitive $\mathrm{K}^{+}$channel subunit $\beta 1$ (KCNMB1) gene. Furthermore, association of the $\beta 1$-regulatory subunit with the $\mathrm{BK}_{\mathrm{Ca}}$ channel increases the $\mathrm{Ca}^{2+}$ sensitivity of the channel and decreases voltage dependence $(8,9)$. Additionally, the single-nucleotide polymorphism (SNP) of the KCNMB1 gene, rs11739136 [which causes protein variation of Glu65Lys (KCNMB1-E65K)], increases the probability of the BK-channel opening with $\mathrm{Ca}^{2+}$ stimulation, enhances $\mathrm{K}^{+}$efflux and membrane hyperpolarization, and reduces artery impedance $(10,11)$.

A previous study on the Han Chinese population demonstrates that a reduced function of $\mathrm{BK}_{\mathrm{Ca}}$ channels with KCNMB1-rs11739136 is associated with EH susceptibility (12). Furthermore, abnormal expression and electrical dysfunction of the adenosine $5^{\prime}$-triphosphate (ATP)-sensitive $\mathrm{K}^{+}$channel $\left(\mathrm{K}_{\mathrm{ATP}}\right)$ is also important in the pathophysiology of cardiovascular diseases $(13,14)$. The $\mathrm{K}_{\mathrm{ATP}}$ channel is an inward rectifier $\mathrm{K}^{+}$channel that is gated by intracellular nucleotides, ATP and adenosine 5'-diphosphate (15). It is widely distributed in excitatory tissues, including skeletal cells, VSMCs and neurons, and non-excitatory tissues including renal tubular epithelial cells and oocytes $(16,17)$. Furthermore, the $\mathrm{K}_{\text {АTP }}$ channel in the smooth muscle cells is composed of the inward-rectifier $\mathrm{K}^{+}$ion channel protein Kir6.2 subunit, the high affinity sulfonylurea receptor (SUR) subunits SUR2B and additional components (15), and is important in the response to stress and changes in blood pressure $(18,19)$. 
The Kir6.2 subunit is important in the function of the $\mathrm{K}_{\mathrm{ATP}}$ channel and is encoded by the $\mathrm{K}^{+}$inwardly rectifying channel, subfamily J, member-11 (KCNJ11) gene (15). Furthermore, the human KCNJ11 gene is located on chromosome 11 p15.1 with only one 1173-bp exon and no introns $(20,21)$. Additionally, the KCNJ11 gene mutations KCNJ11-rs5219 and KCNJ11-rs2285676 are able to alter the polarity of the ATP-binding region and decrease channel sensitivity to ATP that causes dysfunction of the $\mathrm{K}_{\text {ATP }}$ channel $(22,23)$. KCNJ11-rs5219 (KCNJ11-E23K) is a missense mutation located in the amino-terminal of the Kir6.2 subunit, and is associated with cardiovascular diseases and diabetes $(24,25)$. To date, several studies have been performed that demonstrate that the KCNJ11-rs5219 gene polymorphism is significantly associated with EH in Chinese and Korean populations $(26,27)$. However, the association between KCNJ11-rs5219 and $\mathrm{EH}$ is not understood, particularly in Chinese ethnic populations. Therefore, to the best of our knowledge, an association between KCNJ11-rs2285676 gene polymorphism and EH has not yet been reported in Chinese or other populations, as the distribution of genetic polymorphisms is specific to region and ethnicity. Additionally, it has been reported that the prevalence of hypertension in Kazak Chinese populations is significantly higher than that in Uygur and Han Chinese populations in Xinjiang (28).

Based on the information provided above, the aim of the present study was to genotype three SNPs, namely KCNJ11-rs5219 and KCNJ11-rs2285676 of the KCNJ11 gene, and KCNMB1-rs11739136 of the KCNMB1 gene, in a well-defined population of individuals with EH and a healthy control population. This was conducted in order to investigate whether there is an association between the EH-associated $\mathrm{BK}_{\mathrm{Ca}}$ and $\mathrm{K}_{\mathrm{ATP}}$ channel gene polymorphisms and the development of EH in Xinjiang Kazak Chinese populations, and to observe whether they are important genetic risk factors for $\mathrm{EH}$ development in the Kazak Chinese population.

\section{Materials and methods}

Subjects. All Kazak subjects were recruited from Dongwan County of the Shawan Region in Xinjiang, China, between October 2012 and October 2013. Samples were obtained from

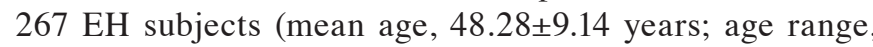
30-75 years; 118 men and 149 women) and 259 normotensive (NT) subjects (mean age, $46.89 \pm 9.60$ years; age range, 30-75 years; 99 men and 160 women), who were selected using the method described in a previous case-control study (29). All patients underwent subjective examinations in the morning following an overnight fast. Blood pressure (BP) measurement was performed in the patient's dominant arm following $>10$ min of rest in a sitting position. A hypertensive status was determined according to blood pressure $>140 / 90 \mathrm{mmHg}$. Furthermore, there was no familial relationship between any subjects. All patients met the criteria of World Health Organization International Society of Hypertension, where hypertension was defined as a systolic blood pressure $(\mathrm{SBP}) \geq 140 \mathrm{mmHg}$ and/or a diastolic blood pressure (DBP) $\geq 90 \mathrm{mmHg}$ with repeated measurements, or receiving anti-hypertensive medication (30). Routine examination and the analysis of various other factors, including gender, obesity, smoking, duration of EH and hypersensitivity to non-steroid anti-inflammatory drugs were performed in order to establish whether subjects with high BP were primary or secondary in nature.

Subjects with secondary hypertension, endocrine diseases (diabetes), or with heart, liver and kidney diseases were excluded based on their medical history or physical examination. The present study was conducted in accordance with the Declaration of Helsinki and with approval from the Institutional Ethics Review Board (IERB) at the First Affiliated Hospital of the Shihezi University School of Medicine (Shihezi, China; IERB no. SHZ2010LL01). Furthermore, written informed consent was obtained from all subjects, prior to participation.

Source of reagents. A whole blood genomic DNA extraction reagent kit (Blood Genome DNA Extraction kit; cat. no. DP348) was acquired from Tiangen Biotech Co., Ltd. (Beijing, China). Hot Start (HS) Taq polymerase (Premix PrimeSTAR HS version; cat. no. DR040A) was obtained from Takara Biotechnology Co., Ltd., (Dalian, China). All restriction enzymes were provided by New England Biolabs, Inc. [cat. nos. R0119 for BanII; R0196 for NciI and R0102 for MbiI (BsrBI); Ipswich, MA, USA]. All primer synthesis and DNA sequencing of the polymerase chain reaction (PCR) products in the present study were performed by Sangon Biotech Co., Ltd. (Shanghai, China).

Biochemical indices. Clinical characteristics of the study subjects are presented in Table I. Peripheral venous blood was obtained from the antecubital vein of the right arm of subjects while they were sitting, and was then placed in EDTA (200 $\mu \mathrm{l})$-coated tubes. Blood was subsequently centrifuged at $1,500 \mathrm{x} \mathrm{g}$ for $10 \mathrm{~min}$ at $25^{\circ} \mathrm{C}$, and then separated plasma was stored at $-80^{\circ} \mathrm{C}$. Triglycerides (TGs), total cholesterol (TC), high-density lipoprotein cholesterol (HDL-C) and low-density lipoprotein cholesterol (LDL-C) were determined using commercially available enzymatic kits (TG kit 4657594190, TC kit 4718917190, HDL-C kit 5401488190 and LDL-C kit 5401682190; Roche Diagnostics, Basel, Switzerland) and a Roche COBAS Integra 800 automated analyzer (Roche Diagnostics).

DNA extraction. DNA was extracted from whole peripheral blood using the rapid whole blood genomic DNA extraction kit according to the manufacturer's instructions. Genomic DNA integrity was analyzed by $1 \%$ agarose gel electrophoresis.

PCR amplification. Primers were designed using Primer Premier 5.0 Software (Premier Biosoft International, Palo Alto, CA, USA) and were synthesized by Sangon Biotech Co., Ltd. PCR amplification of the $\mathrm{K}^{+}$channel gene was performed using the following primers: rs2285676, forward 5'-CAATTC AGGACTGGCTCACC-3' and reverse, 5'-GTAGGCTCCACA GCACCAAC-3'; and rs5219, forward 5'-GACTCTGCAGTG AGGCCCTA-3' and reverse 5'-ACGTTGCAGTTGCCTTTC TT-3'.

As there was no appropriate restriction endonuclease on the KCNMB1-rs11739136 gene, the present study introduced a mismatched base $\mathrm{C}$ on the 27th base of the forward primer according to Zhao et al (12) and to lead into the recognition 
Table I. Clinical and biochemical characteristics of study subjects.

\begin{tabular}{|c|c|c|c|c|}
\hline Parameters analyzed & $\mathrm{EH}(\mathrm{n}=267)$ & NT (n=259) & $\mathrm{T} / \chi^{2}$-value & P-value \\
\hline Age, years & $48.28 \pm 9.14$ & $46.89 \pm 9.60$ & 1.70 & 0.091 \\
\hline Gender, male/female & $118 / 149$ & $99 / 160$ & 1.93 & 0.164 \\
\hline $\mathrm{SBP}, \mathrm{mmHg}$ & $148.75 \pm 19.46$ & $117.85 \pm 11.01$ & 22.34 & $<0.01$ \\
\hline $\mathrm{DBP}, \mathrm{mmHg}$ & $95.67 \pm 12.35$ & $75.19 \pm 7.25$ & 21.03 & $<0.01$ \\
\hline Height, $\mathrm{cm}$ & $163.47 \pm 8.31$ & $161.87 \pm 8.59$ & 2.17 & 0.030 \\
\hline Weight, kg & $70.18 \pm 13.88$ & $65.28 \pm 12.34$ & 4.33 & $<0.01$ \\
\hline $\mathrm{BMI}, \mathrm{kg} / \mathrm{m}^{2}$ & $26.27 \pm 4.50$ & $24.86 \pm 3.84$ & 3.85 & $<0.01$ \\
\hline $\mathrm{TG}, \mathrm{mmol} / \mathrm{l}$ & $1.41 \pm 0.99$ & $1.27 \pm 0.87$ & 1.77 & 0.077 \\
\hline $\mathrm{TC}, \mathrm{mmol} / \mathrm{l}$ & $4.66 \pm 1.20$ & $4.52 \pm 1.03$ & 1.36 & 0.174 \\
\hline LDL-C, mmol/1 & $2.35 \pm 0.80$ & $2.33 \pm 0.72$ & 0.25 & 0.802 \\
\hline HDL-C, mmol/1 & $1.49 \pm 0.50$ & $1.49 \pm 0.42$ & 0.08 & 0.938 \\
\hline
\end{tabular}

Mean \pm standard deviation value for continuous variables. EH, essential hypertension; NT, normaltensive; SBP, systolic blood pressure; DBP, diastolic blood pressure; BMI, body mass index; TG, triglyceride; TC, total cholesterol; LDL-C, low-density lipoprotein cholesterol; HDL-C, high-density lipoprotein cholesterol.
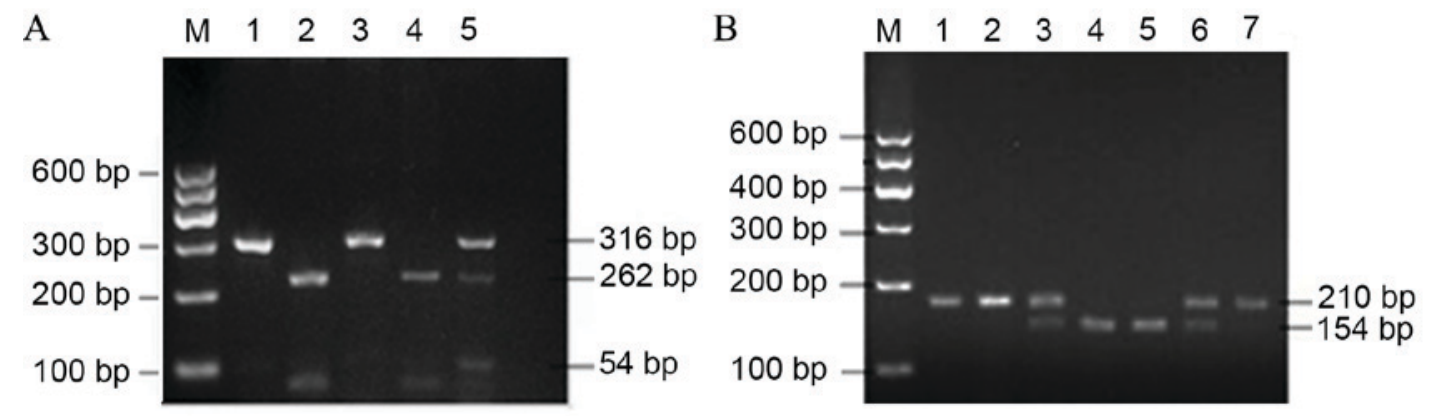

Figure 1. PCR products of KCNJ11-rs2285676 and KCNJ11-rs5219 polymorphisms. (A) PCR products of KCNJ11-rs2285676 were digested by the NciI enzyme. M, marker; 1 and 3, TT genotype; 2 and 4, CC genotype; and 5, CT genotype. (B) PCR products of KCNJ11-rs5219 polymorphisms were digested by the BanII enzyme. M, marker; 4 and 5, GG genotype; 3 and 6, GA genotype; and 1,2 and 7, AA genotype. PCR, polymerase chain reaction; KCNJ11, inwardly rectifying $\mathrm{K}^{+}$channel, subfamily J, member-11.

site of restriction endonuclease $\mathrm{MbiI}$. The forward primer of rs11739136 was 5'-GGTACTGGGGCACCTTCTTGCACT TCCGCT-3', and the reverse primer was 5'-CGGGGACTG TGGGGTCATGTGCCTTT-3'. Amplification was performed using an Eppendorf Mastercycler Gradient 5331 thermocycler (Eppendorf, Hamburg, Germany) in a $25 \mu \mathrm{l}$ volume containing 50 ng DNA, $12.5 \mu 12$ X PrimeSTAR HS [0.05 U/ $\mu 1$ PrimeSTAR HS DNA Polymerase, 2X PrimeSTAR Buffer, $2 \mathrm{mM} \mathrm{MgCl}$ and $2 \mathrm{X}$ dNTP mixture (0.4 mM of each dNTP)], $0.1 \mathrm{M}$ of each primer and double-distilled water. The PCR reaction consisted of 35 cycles of $95^{\circ} \mathrm{C}$ for $5 \mathrm{~min}$, followed by $95^{\circ} \mathrm{C}$ for $30 \mathrm{sec}, 58^{\circ} \mathrm{C}$ for $30 \mathrm{sec}, 72^{\circ} \mathrm{C}$ for $40 \mathrm{sec}$ for $35 \mathrm{cycles}$ and $72^{\circ} \mathrm{C}$ for $8 \mathrm{~min}$. The PCR products were subsequently stored at $4^{\circ} \mathrm{C}$. PCR products were determined by $1 \%$ agarose gel electrophoresis. Following amplification, the lengths of PCR amplified products were 210, 316 and 256 bp for KCNJ11-rs5219, KCNJ11-rs2285676 and KCNMB1-rs11739136, respectively (Figs. 1 and 2).

Restriction enzyme and screening analysis. Digestion was performed using an Eppendorf Mastercycler Gradient 5331 thermocycler (Eppendorf, Hamburg, Germany) in a $30 \mu \mathrm{l}$ volume

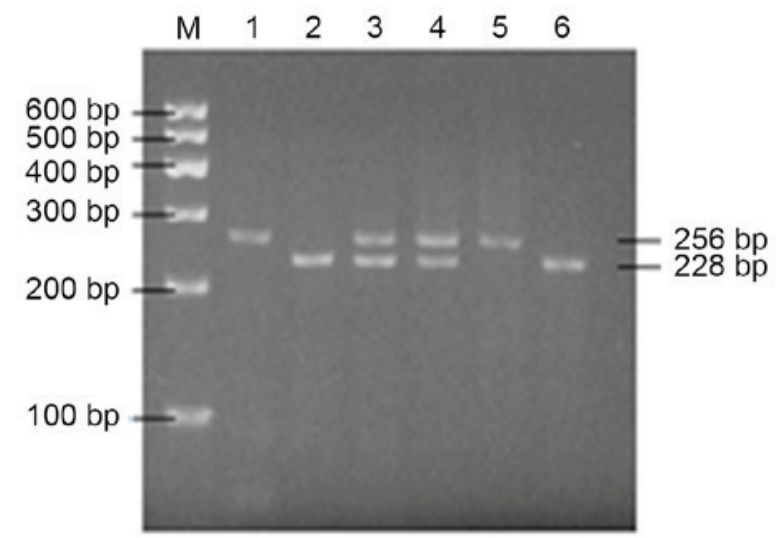

Figure 2. Polymerase chain reaction products of the $\beta 1$ subunit of large-conductance $\mathrm{Ca}^{2+}$-activated $\mathrm{K}^{+}$channel-rs11739136 polymorphism were digested by the MbiI enzyme. M, marker; 1 and 5, TT genotype; 2 and 6, CC genotype; and 3 and 4, CT genotype.

mixture including $10 \mu \mathrm{l}$ PCR product, $16 \mu 1$ nuclease-free water, $3 \mu 1$ 10X CutSmart ${ }^{\circledR}$ Buffer (supplied with the enzyme) 
A

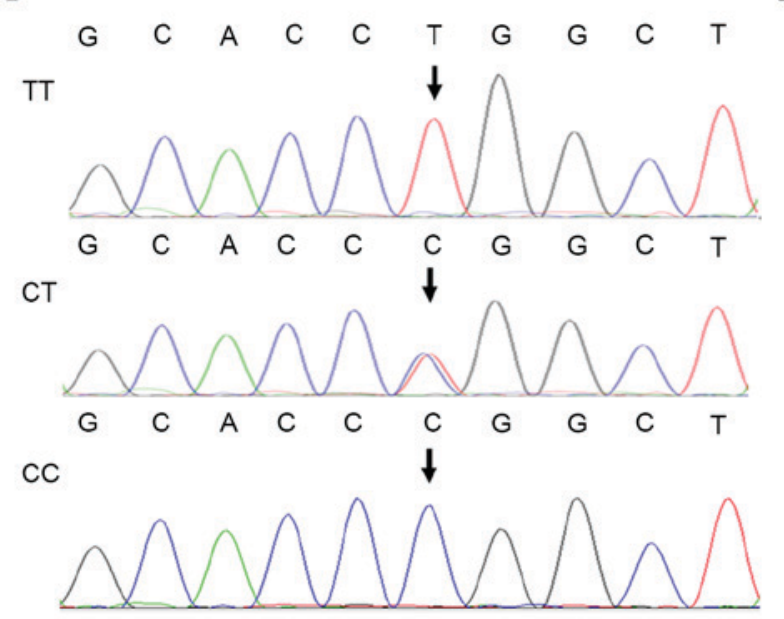

B

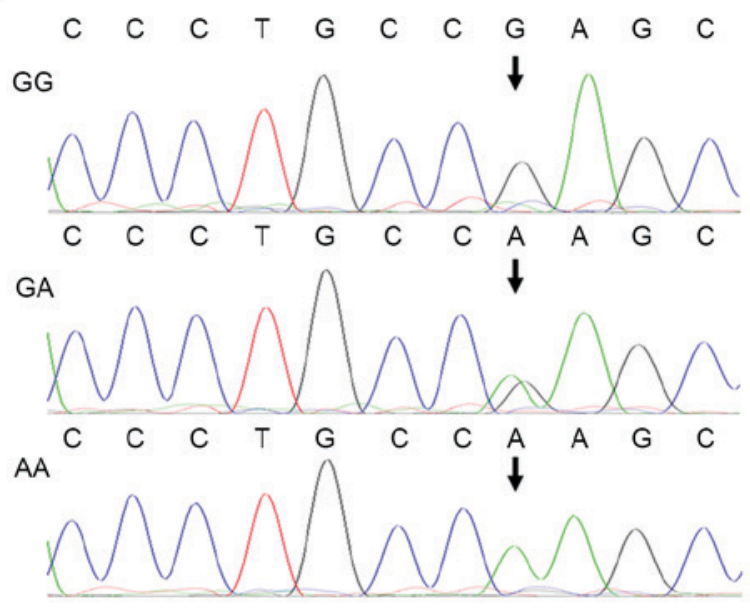

Figure 3. Sequence chromatograms of KCNJ11-rs2285676 and KCNJ11-rs5219 polymorphisms. (A) Sequence chromatograms of inwardly rectifying K channel, subfamily J, member-11 ( KCNJ11)-rs2285676 SNP. (B) Sequence chromatograms of KCNJ11-rs5219 SNP. KCNJ11, inwardly rectifying K+ channel, subfamily J, member-11.

and $1 \mu 1$ restriction enzyme (10 U/ $\mu 1)$. The PCR products of KCNJ11-rs5219, KCNJ11-rs2285676 and KCNMB1-rs11739136 were digested with enzyme BanII, NciI and MbiI, respectively. The $30-\mu \mathrm{l}$ mixtures were incubated at $37^{\circ} \mathrm{C}$ for $3 \mathrm{~h}$. Restricted DNA products were then separated by $2.5 \%$ agarose gel electrophoresis and visualized under UV light. With regard to the SNP KCNJ11-rs2285676 (C/T), a single 316 bp band indicated homozygosity for the T allele (TT); two bands, 262 and $54 \mathrm{bp}$, indicated homozygosity for the $\mathrm{C}$ allele (CC); and the presence of three bands, 316, 262 and $54 \mathrm{bp}$, indicated heterozygosity for the T or C allele (CT) (Fig. 1A). As for the SNP KCNJ11-rs5219 (G/A), the presence of a single $210 \mathrm{bp}$ band indicated homozygosity for the A allele (AA); the presence of two fragments, 154 and $56 \mathrm{bp}$, indicated homozygosity for the $\mathrm{G}$ allele (GG); and three fragments, 210, 154 and 56 bp indicated heterozygosity for the G and A alleles (Fig. 1B). SNP of KCNMB1-rs11739136 through PCR-restriction fragment length polymorphism (PCR-RFLP) experiments generated three types of bands (Fig. 2): One band indicates homozygosity for the $\mathrm{T}$ allele (TT genotype), with a fragment length of $256 \mathrm{bp}$; three bands indicate heterozygosity for the $\mathrm{T}$ or $\mathrm{C}$ allele (CT genotype), with fragment lengths of 256, 228 and $28 \mathrm{bp}$; and two bands indicates homozygosity for the $\mathrm{C}$ allele (CC genotype), with fragment lengths of 228 and $28 \mathrm{bp}$.

The three types of genotypes of KCNJ11-rs2285676 (TT, CT and CC), KCNJ11-rs5219 (AA, GA and GG) and KCNMB1-rs11739136 (TT, CT and CC) following digestion were consistent with the results of DNA sequencing (Figs. 3 and 4). For DNA sequencing of the PCR product, the purified product was sent for Sanger DNA sequencing at Sangon Biotech, Co., Ltd. DNA sequence alignment was analyzed against the KCNJ11 and KCNMB1 reference sequences using ClustalW2 (European Molecular Biology Laboratory, European Bioinformatics Institute, Hinxton, UKs. Additionally, the genotype polymorphism distribution of each group conformed to the Hardy-Weinberg Equilibrium (HWE).

Statistical analysis. Statistical analysis was performed using SPSS 17.0 statistical software (SPSS, Inc., Chicago, IL, USA)

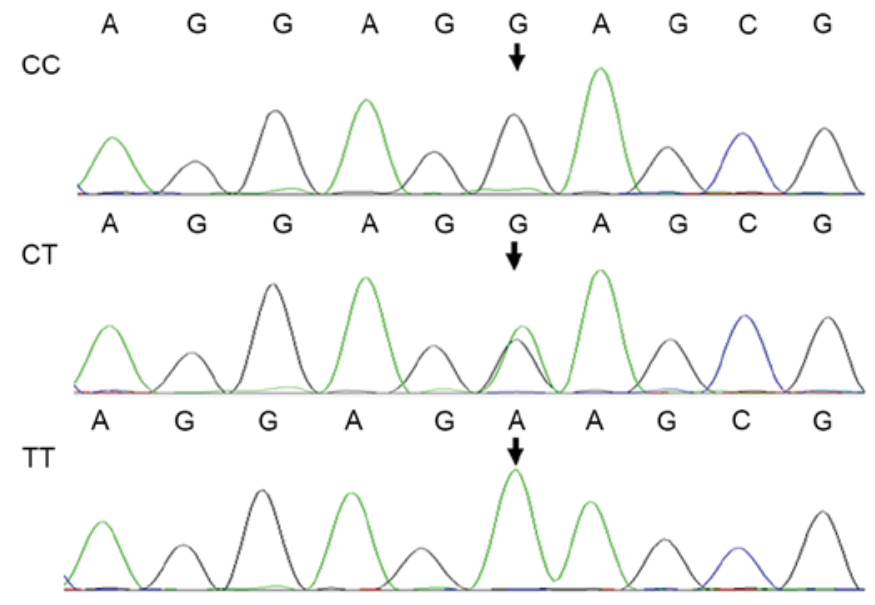

Figure 4. Reverse sequence chromatograms of the $\beta 1$ subunit of large-conductance $\mathrm{Ca}^{2+}$-activated $\mathrm{K}^{+}$channel-rs11739136 polymorphism.

and data for clinical and biochemical characteristics are presented as the mean \pm standard deviation. Comparisons of continuous variables with normal distribution or two groups of mean values between EH and NT groups were performed using an independent Student's t-test. If the data did not conform to a normal distribution, the data were expressed as median and quartiles.

In addition, $\chi^{2}$ analyses was applied to determine the difference in genotype and gene frequency. Unconditional backward stepwise logistic regression adjusted for age, gender and body mass index (BMI) was performed to determine the risk factors of $\mathrm{EH}$ and screen the independent variables. Additionally, hypertension was used as the dependent variable and risk factors associated with hypertension were used as the independent variables. In each model, the odds ratio (OR) for each independent variable with a 95\% confidence interval (CI) was determined from unconditional logistic regression models. For the independent variable, $\mathrm{P}<0.05$ was considered to indicate a statistically significant difference. The frequencies of each polymorphic site were estimated by the allele counting 
method and tested for HWE. Furthermore, HWE was assessed by the $\chi^{2}$ test to compare the observed and expected genotype frequencies among the subjects. $\chi^{2}$ or Fisher's exact tests were used to compare the distribution of genotype and allele frequency between groups. Statistical significance was tested using $\chi^{2}$ and $\mathrm{P}<0.05$ was considered to indicate a statistically significant difference.

\section{Results}

Clinical and biochemical characteristics. In the present study, 267 patients with EH patients (118 males and 149 females) were diagnosed and the mean age of $\mathrm{EH}$ patients was $48.28 \pm 9.14$ years with a mean BMI of $26.27 \pm 4.50 \mathrm{~kg} / \mathrm{m}^{2}$. A total of 259 healthy volunteers (controls; 99 males and 160 females) were also recruited, and their mean age was $46.89 \pm 9.60$ years with a mean BMI of $24.86 \pm 3.84 \mathrm{~kg} / \mathrm{m}^{2}$ (Table I). Table I describes the clinical and biochemical characteristics of the EH patients and controls. A significant difference was observed in the SBP, DBP, height, weight and BMI between hypertensive and NT subjects (all $\mathrm{P}<0.01)$. The mean values of BMI $(\mathrm{t}=3.85, \mathrm{P}<0.01), \mathrm{SBP}(\mathrm{t}=22.34, \mathrm{P}<0.01)$ and $\mathrm{DBP}(\mathrm{t}=21.03$, $\mathrm{P}<0.01)$ were significantly elevated in $\mathrm{EH}$ patients compared with NTs. This indicates that BMI may affect the development of EH. In addition, no significant difference was detected in terms of gender, age, TG, TC, HDL-C and LDL-C between the EH control and NT groups (Table I).

Genotype and allele frequency analysis. To analyze the frequency of the genotypes for tag SNPs in the KCNMB1 and KCNJ11 genes, PCR-RFLP analysis was performed. PCR products corresponding to KCNJ11-rs2285676, KCNJ11-rs5219 and KCNMB1-rs11739136 were digested by NciI, BanII and MbiI restriction endonucleases, respectively. KCNJ11-rs2285676 polymorphisms were categorized into three genotypes: TT (316 bp), CT (316, 262 and 54 bp) and CC (262 and 54 bp) (Fig. 1A). KCNJ11-rs5219 polymorphisms were categorized into AA (210 bp), GA (210, 154 and $56 \mathrm{bp})$ and GG (154 and 56 bp) (Fig. 1B). KCNMB1-rs11739136 polymorphisms were categorized into CC (228 and $28 \mathrm{bp}), \mathrm{CT}$ (256, 228 and $28 \mathrm{bp}$ ) and TT (256 bp) (Fig. 2). It was noted that the 54-, 56-, and 28 bp fragments were too short to be visible. Results of restriction endonuclease analysis were compared with DNA sequencing data and lead to the corresponding results (Figs. 3 and 4 ).

In order to study the association between the genotype and allele frequency distributions in the KCNJ11-rs2285676, KCNJ11-rs5219 and KCNMB1-rs11739136 SNPs, and the risk of $\mathrm{EH}$, the genotype and frequencies of occurrence of the polymorphic forms of KCNJ11-rs2285676, KCNJ11-rs5219 and KCNMB1-rs11739136 was analyzed. The distribution of the genotype frequencies of KCNJ11-rs5219, KCNJ11-rs2285676 and KCNMB1-rs11739136 in the EH and NT groups is in accordance with the HWE.

Three $\mathrm{SNP}_{\mathrm{S}}$ were genotyped in 526 individuals of the overall sample. As shown in Table II, for the KCNJ11-rs2285676 $\mathrm{SNP}$, the frequency of the CC and (CT+TT) genotypes was 6.95 and $93.05 \%$ in the controls, and 4.12 and $95.88 \%$ in patients, respectively. Furthermore, there was no statistically significant difference in the genotype frequencies [CC vs. $\left.\mathrm{CT}+\mathrm{TT}, \mathrm{OR}=0.58(95 \% \mathrm{CI}, 0.27-1.24) ; \chi^{2}=2.02 ; \mathrm{P}=0.155\right]$ and allele frequencies [C vs. T, OR=1.06 (95\% CI, 0.81-1.39); $\mathrm{P}=0.655$ ] distribution between the EH and NT groups. However, for KCNJ11-rs5219, a significantly higher frequency of the rs5219 polymorphism GG genotype was shown in the EH patients group compared with the control group (34.83 vs. $20.46 \%$, respectively; $\mathrm{P}<0.01)$. Furthermore, a significantly lower frequency of the KCNJ11-rs5219 polymorphism $\mathrm{GA}+\mathrm{AA}$ genotype was observed in the group of $\mathrm{EH}$ patients in comparison with the healthy population (65.17 vs. $79.54 \%$; $\mathrm{P}<0.01)$. The frequencies of KCNJ11-rs5219 $\mathrm{G}$ and $\mathrm{A}$ alleles in the EH group were 61.42 and $38.58 \%$, respectively. Finally, the KCNJ11-rs5219 $\mathrm{G}$ allele distribution of the EH group was significantly higher than that of the NT group [G vs. A, OR, 1.28 (95\% CI, 1.00-1.64); $\left.\chi^{2}=3.92 ; \mathrm{P}=0.048\right]$. Relative risk analysis demonstrated that subjects carrying the rs5219 GG genotype were at 2.08 times greater risk to have $\mathrm{EH}$ than the (GA+AA) genotype carriers (95\% CI, 1.40-3.08).

The KCNMB1-rs11739136 variant in EH patients of Xinjiang Kazak Chinese origin was also assessed, as shown in Table II. The genotype frequencies for CC and CT+TT in EH patients vs. NT subjects were 70.04 and $29.96 \%$ vs. 63.71 and $36.29 \%$. Furthermore, there were no statistically significant differences in the genotypic distribution and the allelic frequency between EH and NT subjects [CC vs. CT+TT, $\mathrm{OR}=1.33$ (95\% CI, 0.93-1.92); $\mathrm{P}=0.123$, and $\mathrm{C}$ vs. $\mathrm{T}, \mathrm{OR}=1.31$ (95\% CI, 0.96-1.78); $\mathrm{P}=0.085]$.

Logistic regression analysis. As the GG genotype of the KCNJ11-rs5219 SNP in the EH group was significantly higher than that of the NT group, the present study assessed the association between the GG genotype and the risk of EH by logistic regression analysis after adjusting for BMI. This individual polymorphism combined with BMI were revealed to be significantly associated with EH $(\mathrm{P}<0.01$; Table III). Therefore, in the BMI-adjusted model GG genotype [OR=2.15 (95\% CI, 1.44-3.21); $\mathrm{P}<0.05]$ and BMI [OR=1.09 (1.04-1.1365), $\mathrm{P}<0.01]$ increased the risk of $\mathrm{EH}$ in Xinjiang Kazak Chinese individuals.

\section{Discussion}

$\mathrm{EH}$ is a complex cardiovascular disease caused by the interaction of polygenic inheritance with environmental risk factors. The Kazak population from Xinjiang has a relatively unique lifestyle, which includes eating more animal fat, a high protein and high-salt diet, and lower consumption of fresh fruit and vegetables (31). Furthermore, the genetic background of the Kazak population is distinct from the Han population, and may lead to differences in genes and diseases caused by genetic susceptibility, including hypertension (31). The genetic variants studied in the present study are useful in the validation of individuals that have a high risk for $\mathrm{EH}$, and this may provide useful information for disease diagnosis and prevention. In the present study, the SBP, DBP and BMI were significantly different between NT subjects and EH patients, indicating that the general clinical data were significantly different between Kazak EH patients and the Kazak NT group. In the group of EH patients, SBP and DBP were higher than that of the patients from the NT group and, therefore, 
Table II. Genotype and allele frequencies of inwardly rectifying $\mathrm{K}^{+}$channel, subfamily $\mathrm{J}$, member-11 and $\beta 1$ subunit of large-conductance $\mathrm{Ca}^{2+}$-activated $\mathrm{K}^{+}$channel SNPs.

\begin{tabular}{|c|c|c|c|c|c|c|c|}
\hline SNP & Gene & $\mathrm{EH}(\mathrm{n}=267)$ & NT $(\mathrm{n}=259)$ & P-value & $\chi^{2}$ & OR & $95 \% \mathrm{CI}$ \\
\hline \multirow[t]{8}{*}{ rs2285676 } & Genotype & & & & & & \\
\hline & $\mathrm{CC}$ & $11(4.12 \%)$ & $18(6.95 \%)$ & & & & \\
\hline & $\mathrm{CT}$ & $131(49.06 \%)$ & $106(40.93 \%)$ & & & & \\
\hline & TT & $125(46.82 \%)$ & $135(52.12 \%)$ & & & & \\
\hline & $\mathrm{CT}+\mathrm{TT}$ & $256(95.88 \%)$ & $241(93.05 \%)$ & 0.155 & 2.02 & 0.58 & $0.27-1.24$ \\
\hline & Allele & & & & & & \\
\hline & C & $153(28.65 \%)$ & $142(27.41 \%)$ & & & & \\
\hline & $\mathrm{T}$ & $381(71.35 \%)$ & $376(72.59 \%)$ & 0.655 & 0.20 & 1.06 & $0.81-1.39$ \\
\hline \multirow[t]{8}{*}{ rs5219 } & Genotype & & & & & & \\
\hline & GG & $93(34.83 \%)$ & $53(20.46 \%)$ & & & & \\
\hline & GA & $142(53.18 \%)$ & $181(69.88 \%)$ & & & & \\
\hline & AA & $32(11.99 \%)$ & $25(9.66 \%)$ & & & & \\
\hline & $\mathrm{GA}+\mathrm{AA}$ & $174(65.17 \%)$ & $206(79.54 \%)$ & $<0.01$ & 13.54 & 2.08 & $1.40-3.08$ \\
\hline & Allele & & & & & & \\
\hline & G & $328(61.42 \%)$ & $287(55.41 \%)$ & & & & \\
\hline & $\mathrm{A}$ & $206(38.58 \%)$ & $231(44.59 \%)$ & 0.048 & 3.92 & 1.28 & $1.00-1.64$ \\
\hline \multirow[t]{8}{*}{ rs11739136 } & Genotype & & & & & & \\
\hline & $\mathrm{CC}$ & $187(70.04 \%)$ & $165(63.71 \%)$ & & & & \\
\hline & $\mathrm{CT}$ & $67(25.09 \%)$ & $76(29.34 \%)$ & & & & \\
\hline & $\mathrm{TT}$ & $13(4.87 \%)$ & $18(6.95 \%)$ & & & & \\
\hline & $\mathrm{CT}+\mathrm{TT}$ & $80(29.96 \%)$ & $94(36.29 \%)$ & 0.123 & 2.38 & 1.33 & $0.93-1.92$ \\
\hline & Allele & & & & & & \\
\hline & $\mathrm{C}$ & 441 (82.58\%) & 406 (78.38\%) & & & & \\
\hline & $\mathrm{T}$ & $93(17.42 \%)$ & $112(21.62 \%)$ & 0.085 & 2.97 & 1.31 & $0.96-1.78$ \\
\hline
\end{tabular}

SNP, single nucleotide polymorphism; EH, essential hypertension; NT, normaltensive; OR, odds ratio; CI, confidence interval.

Table III. Multivariate logistic regression analysis of hypertension in Xinjiang Kazak.

\begin{tabular}{lcccccc}
\hline Factors & B & SE & Walds & P-value & OR value & 95\% CI \\
\hline BMI & 0.084 & 0.022 & 14.719 & $<0.01$ & 1.09 & $1.04-1.1365$ \\
rs5219 GG Genotype & 0.765 & 0.204 & 14.073 & $<0.01$ & 2.15 & $1.44-3.21$ \\
\hline
\end{tabular}

BMI, body mass index; SE, standard error; OR, odds ratio; CI, confidence interval.

may contribute to increased mortality from cardiovascular pathology (32). The mean BMI of Kazak EH patients was significantly greater than that of NT controls, which may result from the high-fat diet of the Kazak population. This result suggested that hypertension is associated with lipid metabolism.

As there are substantial ethnic and regional differences in the distribution of gene polymorphisms, a Kazak Chinese population in Xinjiang was selected as the research cohort. Kazak Chinese individuals have minimal population migration and geographical environment separation, and similar genetic backgrounds; these features benefitted the study of the association between KCNJ11 and KCNMB1 gene polymorphism and EH. In the present case-control study, the association of KCNJ11 (rs5219 and rs2285676) and KCNMB1 (rs11739136) gene polymorphisms with EH was explored in a Kazak population. Previous studies have demonstrated that there is an association between different KCNJ11 SNPs and $\mathrm{EH}$ and diabetes in different populations (33-35). However, to the best of our knowledge, there are no studies that have been performed with these SNPs in the Kazak population. In an animal model of hypertension, knocking out the KCNJ11 gene has been demonstrated to induce heart failure and mortality (36). Therefore, the KCNJ11-encoded $\mathrm{K}_{\text {ATP }}$ channel is essential to prevent hypertension, with channel alteration being a molecular basis for $\mathrm{EH}$. 
The polymorphic loci in KCNJ11 E23K or rs5219g.67 (G>A) are associated with the risk of hypertension as well as the risk of type 2 diabetes mellitus (T2DM) in the Korean population (27). In addition, Yi et al (14) have previously reported that the EE (E:Glu) genotype of KCNJ11-E23K (rs5219) was associated with body weight, $\mathrm{BMI}, \mathrm{VO}_{2}$ max and maximal minute ventilation in elderly females, as well as DBP in men at rest. Previously, a meta-analysis study demonstrated that the KCNJ11-rs5219 genotype is independently associated with hypertension in East Asian populations (37). Zhuang et al (38) further demonstrated that the SBP of homozygote GG individuals is higher than that of homozygous AA individuals, and the GG genotype increases the risk for hypertension in the Han Chinese population. Consistent with these previous studies, the present study also demonstrated that the frequency of the GG (rs5219) genotype and allele of the KCNJ11-rs5219 SNP site was significantly different between Kazak EH patients and NT controls. The relative risk analysis demonstrated that the individuals carrying the rs5219-GG genotype have a greater risk of developing EH than those individuals carrying the GA+AA genotype. This may be caused by a missense mutation of KCNJ11-rs5219, which would lead to glutamic acid (E) replaced by lysine $(\mathrm{K})$, which would thereby reduce $\mathrm{K}_{\mathrm{ATP}}$ channel sensitivity to ATP and induce $\mathrm{K}_{\mathrm{ATP}}$ channel excessive activation, enhance $\mathrm{K}^{+}$efflux and membrane hyperpolarization, and cause vasodilation $(39,40)$. Therefore, genetic E23K variants which determine open state probability also determine vascular tone and consequently affect blood pressure. Furthermore, in a sample of this size, the single SNP and $\mathrm{EH}$ associations are statistically distinguishable therefore it may be determined that the signal is arising from this SNP where it sits. Therefore, the GG (rs5219) genotype may increase the risk of $\mathrm{EH}$ in the Kazak population.

At present, the correlation between KCNJ11-rs2285676 SNPs and hypertension has not been studied extensively. However, the KCNJ11-rs2285676 polymorphism has been demonstrated to be associated with T2DM in Japanese and Korean populations, as well as in a Chinese Han population (41). A recent study found that the T2DM patients that benefitted from dipeptidyl peptidase-4 inhibitor treatment were those with a KCNJ11 rs2285676 (genotype CC) polymorphism and DBP values within normal ranges (41). The present study's data of risk factor analysis suggests that KCNJ11-rs2285676 genotypes were not associated with EH. Whether the KCNJ11-rs2285676 SNPs are associated with $\mathrm{EH}$ requires further study. $\mathrm{BK}_{\mathrm{Ca}}$ channel $\beta$-subunits have four different subtypes ( $\beta 1-4)$, and the $\beta 1$-subunit is predominantly expressed in the smooth muscle cell (42). It regulates the activity of the $\mathrm{BK}_{\mathrm{Ca}}$ channel and increases vasodilatation $(43,44)$. Furthermore, it has been reported that reduction or deletion of the $\beta 1$ subunit causes membrane depolarization and vascular smooth muscle contraction, which results in cardiovascular disorders, including hypertension and heart failure $(45,46)$.

Gain-of-function mutation of the $\beta 1$-subunit KCNMB1 (rs11739136-Glu65Lys mutant) was caused by a single nucleotide substitution in exon-3 of KCNMB1 (47). The correlation analysis of the KCNMB1-rs11739136 gene mutation and hypertension revealed that the rs11739136 mutation increased $\mathrm{BK}_{\mathrm{Ca}}$ channel sensitivity to $\mathrm{Ca}^{2+}$, which makes blood vessels easier to dilate (47). Nielsen et al (11) reported the KCNMB1
Glu65Lys (rs11739136) polymorphism is associated with reduced SBP and DBP in middle-aged Danish men. In addition, Zhao et al (12) demonstrated that the TT+CT genotype of KCNMB1-rs11739136 had a significantly decreased risk for hypertension. In the present study, the frequency analysis demonstrated that there was no significant difference in the genotype and allele frequency of KCNMB1-rs11739136 between the EH and NT groups. This demonstrates that the SNP sites of KCNMB1-rs11739136 are not associated with the development of EH in the Kazak population. Additionally, the association between KCNMB1-rs11739136 and blood pressure regulation is not affected by age, gender and anti-hypertensive therapy. Therefore, the $\mathrm{T}$ allele is a protective factor that decreases the prevalence of hypertension in the Chinese Han population (12).

In conclusion, the presence of the $\mathrm{G}$ nucleotide at position g.67 (G>A) of KCNJ11-rs5219 is associated with EH in Chinese Kazak populations in Xinjiang, therefore the higher frequency of A allele in EH cases compared with NT may be a genetic susceptibility factor for the development of EH. Therefore, polymorphisms of KCNJ11-rs5219 may be important in EH susceptibility. However, greater sample sizes are required to confirm this observation and also to identify additional populations. The results of the present study provide a basis for developing novel strategies for the diagnosis and treatment of $\mathrm{EH}$ in the Kazak population.

\section{Acknowledgements}

The present study was supported by grants from the National Basic Research Program of China (grant no. 2012CB52660000) and the National Natural Science Foundation of China (grant nos. 81560081, 31460264 and 81560175).

\section{References}

1. Köhler R: Single-nucleotide polymorphisms in vascular $\mathrm{Ca}^{2+}$-activated $\mathrm{K}^{+}$-channel genes and cardiovascular disease. Pflugers Arch 460: 343-351, 2010.

2. Doris PA: Hypertension genetics, single nucleotide polymorphisms, and the common disease: Common variant hypothesis. Hypertension 39: 323-331, 2002.

3. Folkow B: Physiological aspects of primary hypertension. Physiol Rev 62: 347-504, 1982.

4. Li L, Wang R, Ma KT, Li XZ, Zhang CL, Liu WD, Zhao L and Si JQ: Differential effect of calcium-activated potassium and chloride channels on rat basilar artery vasomotion. J Huazhong Univ Sci Technolog Med Sci 34: 482-490, 2014.

5. Ma KT, Li XZ, Li L, Jiang XW, Chen XY, Liu WD, Zhao L, Zhang ZS and Si JQ: Role of gap junctions in the contractile response to agonists in the mesenteric artery of spontaneously hypertensive rats. Hypertens Res 37: 110-115, 2014.

6. Yi F, Wang H, Chai Q, Wang X, Shen WK, Willis MS, Lee HC and $\mathrm{Lu}$ T: Regulation of large conductance $\mathrm{Ca} 2+$-activated $\mathrm{K}+$ (BK) channel $\beta 1$ subunit expression by muscle RING finger protein 1 in diabetic vessels. J Biol Chem 289: 10853-10864, 2014.

7. Grimm PR, Irsik DL, Settles DC, Holtzclaw JD and Sansom SC: Hypertension of Kcnmb1-/- is linked to deficient K secretion and aldosteronism. Proc Natl Acad Sci USA 106: 11800-11805, 2009.

8. Jiang Z, Wallner M, Meera P and Toro L: Human and rodent MaxiK channel beta-subunit genes: Cloning and characterization. Genomics 55: 57-67, 1999.

9. Xu H, Garver H, Galligan JJ and Fink GD: Large-conductance $\mathrm{Ca} 2+$-activated $\mathrm{K}+$ channel beta1-subunit knockout mice are not hypertensive. Am J Physiol Heart Circ Physiol 300: H476-H485, 2011. 
10. Kelley-Hedgepeth A, Peter I, Montefusco MC, Levy D, Benjamin EJ, Vasan RS, Mendelsohn ME, Housman D, Huggins GS and Mitchell GF: The KCNMB1 E65K variant is associated with reduced central pulse pressure in the community-based Framingham Offspring Cohort. J Hypertens 27: 55-60, 2009.

11. Nielsen T, Burgdorf KS, Grarup N, Borch-Johnsen K, Hansen T, Jørgensen T, Pedersen O and Andersen G: The KCNMB1 Glu65Lys polymorphism associates with reduced systolic and diastolic blood pressure in the Inter99 study of 5729 Danes. J Hypertens 26: 2142-2146, 2008.

12. Zhao Q, Wang L, Yang W, Chen S, Huang J, Fan Z, Li H, Lu X and $\mathrm{Gu} \mathrm{D}$ : Interactions among genetic variants from contractile pathway of vascular smooth muscle cell in essential hypertension susceptibility of Chinese Han population. Pharmacogenet Genomics 18: 459-466, 2008.

13. Seino S and Miki T: Physiological and pathophysiological roles of ATP-sensitive K+ channels. Prog Biophys Mol Biol 81: 133-176, 2003.

14. Yi Y, Dongmei L, Phares DA, Weiss EP, Brandauer J and Hagberg JM: Association between KCNJ11 E23K genotype and cardiovascular and glucose metabolism phenotypes in older men and women. Exp Physiol 93: 95-103, 2008.

15. Haghvirdizadeh P, Mohamed Z, Abdullah NA, Haghvirdizadeh P, Haerian MS and Haerian BS: KCNJ11: Genetic Polymorphisms and Risk of Diabetes Mellitus. J Diabetes Res 2015: 908152, 2015.

16. Duan R, Cui W and Wang H: Mutational analysis of the Kir6.1 gene in Chinese hypertensive patients treated with the novel ATP-sensitive potassium channel opener iptakalim. Exp Ther Med 2: 757-760, 2011.

17. Banas K, Clow C, Jasmin BJ and Renaud JM: The KATP channel Kir6.2 subunit content is higher in glycolytic than oxidative skeletal muscle fibers. Am J Physiol Regul Integr Comp Physiol 301: R916-R925, 2011.

18. Haider S, Antcliff JF, Proks P, Sansom MS and Ashcroft FM: Focus on Kir6.2: A key component of the ATP-sensitive potassium channel. J Mol Cell Cardiol 38: 927-936, 2005.

19. Chowdhury UR, Holman BH and Fautsch MP: ATP-sensitive potassium (K(ATP)) channel openers diazoxide and nicorandil lower intraocular pressure in vivo. Invest Ophthalmol Vis Sci 54: 4892-4899, 2013.

20. Duan YH and Chen FX: Structure, function and modulation of $\mathrm{K}_{\text {AтP }}$ channels. Journal of Medical Molecular Biology 4: 2007.

21. Hibino H, Inanobe A, Furutani K, Murakami S, Findlay I and Kurachi Y: Inwardly rectifying potassium channels: Their structure, function, and physiological roles. Physiol Rev 90: 291-366, 2010.

22. Xia XH, Yang $\mathrm{AH}$ and $\mathrm{Hu} \mathrm{Y}$ : Effects of E23K polymorphism in KCNJ11 gene on membrane current. Zhongguo Ying Yong Sheng Li Xue Za Zhi 30: 23-26, 2014 (In Chinese).

23. Jeron A, Hengstenberg C, Holmer S, Wollnik B, Riegger GA, Schunkert $\mathrm{H}$ and Erdmann J: KCNJ11 polymorphisms and sudden cardic death in patient with acute myocardial infarction. J Mol Cell Cardiol 36: 287-293, 2004

24. Xi HL, Liu JF, Li L and Wan J: Relationship between dilated cardiomyopathy and the $\mathrm{E} 23 \mathrm{~K}$ and $\mathrm{I} 337 \mathrm{~V}$ polymorphisms in the Kir6.2 subunit of the KATP channel. Genet Mol Res 12: 4383-4392, 2013.

25. Qin LJ, Lv Y and Huang QY: Meta-analysis of association of common variants in the KCNJ11-ABCC8 region with type 2 diabetes. Genet Mol Res 12: 2990-3002, 2013.

26. Li JY, Li ZB, Zhu M, Liu YQ, Li Y, Wang SW and Zhu QL: Mutational analysis of KCNJ11 in Chinese elderly essential hypertensive patients. J Geriatr Cardiol 9: 153-157, 2012.

27. Koo BK, Cho YM, Park BL, Cheong HS, Shin HD, Jang HC Kim SY, Lee HK and Park KS: Polymorphisms of KCNJ11 (Kir6.2 gene) are associated with Type 2 diabetes and hypertension in the Korean population. Diabet Med 24: 178-186, 2007.

28. Ma XJ, Zhang M, Guo SX, Ma RL, Ding YS, Guo H, Zhang JQ, Niu Q, Liu JM, Li SG, et al: Prevalence of hypertension in Uygur, Kazakhs, and Han people in rural areas of Xinjiang. Chin J Hypertension 21: 1164-1168, 2013.

29. He F, Zhao D, Deng F, Zhong H, Shi X, Yang J, Guo S, Cheng J, Huang G, Tang B, et al: Association of TGF-betal gene polymorphisms in exon1 and blood levels with essential hypertension. Blood Press 19: 225-233, 2010.
30. Whitworth JA; World Health Organization, International Society of Hypertension Writing Group: 2003 World Health Organization (WHO)/International Society of Hypertension (ISH) statement on management of hypertension. J Hypertens 21: 1983-1992, 2003.

31. Li N, Wang H, Yan Z, Yao X, Hong J and Zhou L: Ethnic disparities in the clustering of risk factors for cardiovascular disease among the Kazakh, Uygur, Mongolian and Han populations of Xiniiang: A cross-sectional study. BMC Public Health 12: 499, 2012.

32. Grygiel-Górniak B, Kaczmarek E, Mosor M, Przysławski J and Nowak J: Association of PPAR- $\gamma 2$ and $\beta 3$-AR Polymorphisms With Postmenopausal Hypertension. J Clin Hypertens (Greenwich) 17: 549-556, 2015

33. Wang Y, Zhou XO, Zhang Y, Gao PJ and Zhu DL: Association of KCNJ11 with impaired glucose regulation in essential hypertension. Genet Mol Res 10: 1111-1119, 2011.

34. Khan IA, Vattam KK, Jahan P, Mukkavli KK, Hasan Q and Rao P: Correlation between KCNQ1 and KCNJ11, gene polymorphisms and type 2 and post-transplant diabetes mellitus in the Asian Indian population. Genes Diseases 2: 276-282, 2015.

35. Jamaluddin JL, Huri HZ and Vethakkan SR: Clinical and genetic predictors of dipeptidyl peptidase- 4 inhibitor treatment response in Type 2 diabetes mellitus. Pharmacogenomics 17: 867-881, 2016

36. Kane GC, Behfar A, Dyer RB, O'Cochlain DF, Liu XK, Hodgson DM, Reyes S, Miki T, Seino S and Terzic A: KCNJ11 gene knockout of the Kir6.2 KATP channel causes maladaptive remodeling and heart failure in hypertension. Hum Mol Genet 15: 2285-2297, 2006

37. Sakamoto Y, Inoue H, Keshavarz P, Miyawaki K, Yamaguchi Y, Moritani M, Kunika K, Nakamura N, Yoshikawa T, Yasui N, et al: SNPs in the KCNJ11-ABCC8 gene locus are associated with type 2 diabetes and blood pressure levels in the Japanese population. J Hum Genet 52: 781-793, 2007.

38. Zhuang L, Zhao Y, Zhao W, Li M, Yu M, Lu M, Zhang R, Ge X, Zheng T, Li C, et al: The E23K and A190A variations of the KCNJ11 gene are associated with early-onset type 2 diabetes and blood pressure in the Chinese population. Mol Cell Biochem 404: 133-141, 2015.

39. Kawano N, Emoto M, Mori K, Yamazaki Y, Urata H, Tsuchikura S, Motoyama K, Morioka T, Fukumoto S, Shoji T, et al: Association of endothelial and vascular smooth muscle dysfunction with cardiovascular risk factors, vascular complications and subclinical carotid atherosclerosis in type 2 diabetic patients. J Atheroscler Thromb 19: 276-284, 2012.

40. Schwanstecher C, Meyer U and Schwanstecher M: K(IR)6.2 polymorphism predisposes to type 2 diabetes by inducing overactivity of pancreatic beta-cell ATP-sensitive $\mathrm{K}(+)$ channels. Diabetes 51: 875-879, 2002

41. Jamaluddin JL, Huri HZ and Vethakkan SR: Clinical and genetic predictors of dipeptidyl peptidase-4 inhibitor treatment response in Type 2 diabetes mellitus. Pharmacogenomics 17: 867-881, 2016.

42. Tanaka Y, Meera P, Song M, Knaus HG and Toro L: Molecular constitutents of maxi $\mathrm{KCa}$ channels in human coronary smooth muscle: Predominat alpha + beta subunit complexes. J Physiol 502: 545-557, 1997.

43. Gruslova A, Semenov I and Wang B: An extracellular domain of the accessory $\beta 1$ subunit is required for modulating BK channel voltage sensor and gate. J Gen Physiol 139: 57-67, 2012.

44. Sweet TB and Cox DH: Measuring the influence of the $\mathrm{BKCa}$ \{beta\}1 subunit on $\mathrm{Ca} 2+$ binding to the BKCa channel. J Gen Physiol 133: 139-150, 2009.

45. Amberg GC and Santana LF: Down regulation of the BK channel beta1 subunit in genetic hypertension. Circ Res 93: 965-971, 2003.

46. Wan E, Kushner JS, Zakharov S, Nui XW, Chudasama N, Kelly C, Waase M,Doshi D, Liu G, Iwata S, et al: Reduced vascular smooth muscle BK channel current underlies heart failure-induced vasoconstriction in mice. FASEB J 27: 1859-1867, 2013.

47. Fernández-Fernández JM, Tomás M,VázquezE, OrioP,Latorre R, Sentí M, Marrugat J and Valverde MA: Gain-of-function mutation in the KCNMB1 potassium channel subunit is associated with low prevalence of diastolic hypertension. J Clin Invest 113: 1032-1039, 2004 